\title{
Use of the Bipedicled Scalp Flap for Forehead Reconstruction
}

JOHN G. STONER, M.D.

NEIL A. SWANSON, M.D.

Surgical defects following Mohs surgery can present challenging reconstructive problems. Defect size, shape, and depth cannot be predetermined; and consequently, repairs cannot be preplanned. As a result, the reconstructive surgeon, when designing a cosmetic repair, has to be both ingenious and resourceful. Multiple factors must be considered, including lines of minimal tension, innervation, vascularity, tissue movability, and elasticity. Skin of the upper forehead and scalp, although movable, is as elastic as other facial skin, for example, the cheek. Due to the inherent inelasticity of this skin, closure of forehead defects can require a great deal of tissue movement in order to minimize tension along the suture line. Suture lines running contrary to normal skin lines as a result of rotation or transposition flaps may result in poor cosmetic scars. Although several options for repair of forehead defects exist, we have found this flap to be simple and useful. The forehead-releasing incision creates a bipedicle advancement flap designed to (1) create movable forehead and scalp skin, (2) maintain an excellent blood supply, (3) create a secondary defect that can be hidden well within the hairline of the scalp, and (4) allow for a linear closure of the primary defect within or paralleling existing skin lines. A series of three cases is reported.

John G. Stoner, M.D., is Clinical Assistant Professor of Dermatology, Johns Hopkins, Baltimore, Maryland, and he is in private practice in York, Pennsylvania.

Neil A. Swanson, M.D., is Assistant Professor, Departments of Dermatology and Otolaryngology, University of Michigan Medical Center, Ann Arbor, Michigan.

Address reprint requests to Neil A. Swanson, M.D., C2074 Outpatient Building, Department of Dermatology, University of Michigan, Ann Arbor, MI 48109.

J. Dermatol. Surg. Oncol. 10:3 March 1984

\section{CASE REPORTS}

Case 1

A 70-year-old white female presented with a $1.5 \times$ $1.5 \mathrm{~cm}$ recurrent basal-cell carcinoma on the left upper forehead. This lesion was excised by Mohs surgery fresh-tissue technique in two stages, with a resultant defect measuring $4 \times 2 \mathrm{~cm}$. The wound was closed with a bipedicle forehead-releasing flap.

\section{Case 2}

A 64-year-old white female presented with a $1.0 \times$ $1.0 \mathrm{~cm}$ recurrent basal-cell carcinoma on the central upper forehead. Following a three-stage Mohs surgical procedure, she was left with a $2.5 \times 1.5 \mathrm{~cm}$ defect, which was closed by creating a bipedicle advancement flap.

\section{Case 3}

An 84-year-old white female presented with a 1.5 $\times 1.2 \mathrm{~cm}$ recurrent basal-cell carcinoma of the central upper forehead. The lesion was excised by Mohs surgery in two stages, leaving a $2 \times 2 \mathrm{~cm}$ defect. A relaxing incision within the scalp created a bipedicle advancement flap to cover this defect.

\section{DESIGN AND MOVEMENT OF THE FLAP}

After tumor extirpation, the length and width of the primary defect are measured (Fig. 1). After undermining, the flap is incised linearly within the hairline at a distance equal to or slightly greater than 


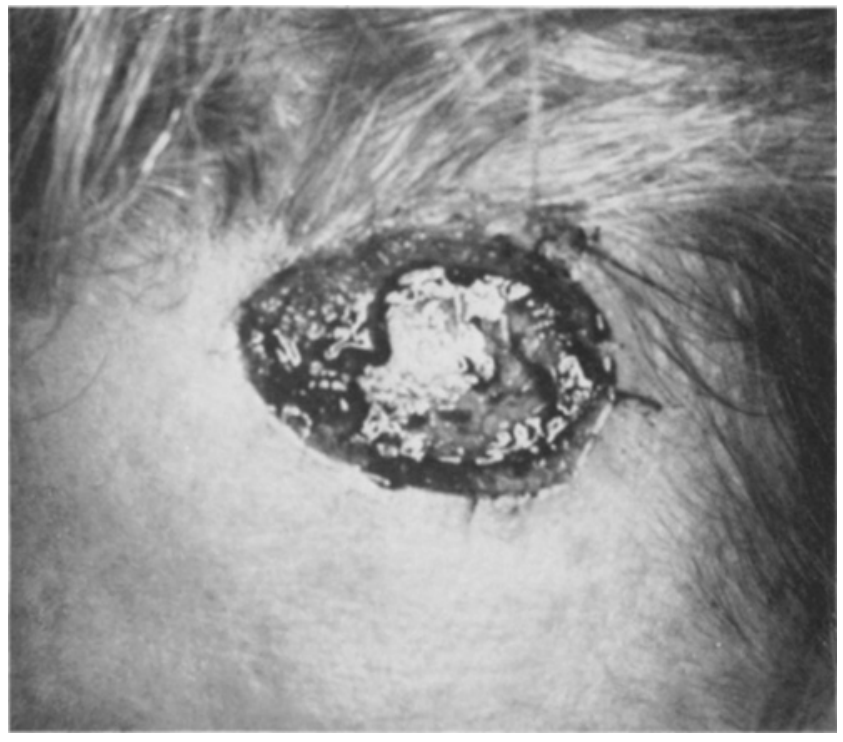

FIGURE 1. Length and width of the primary defect are measured.

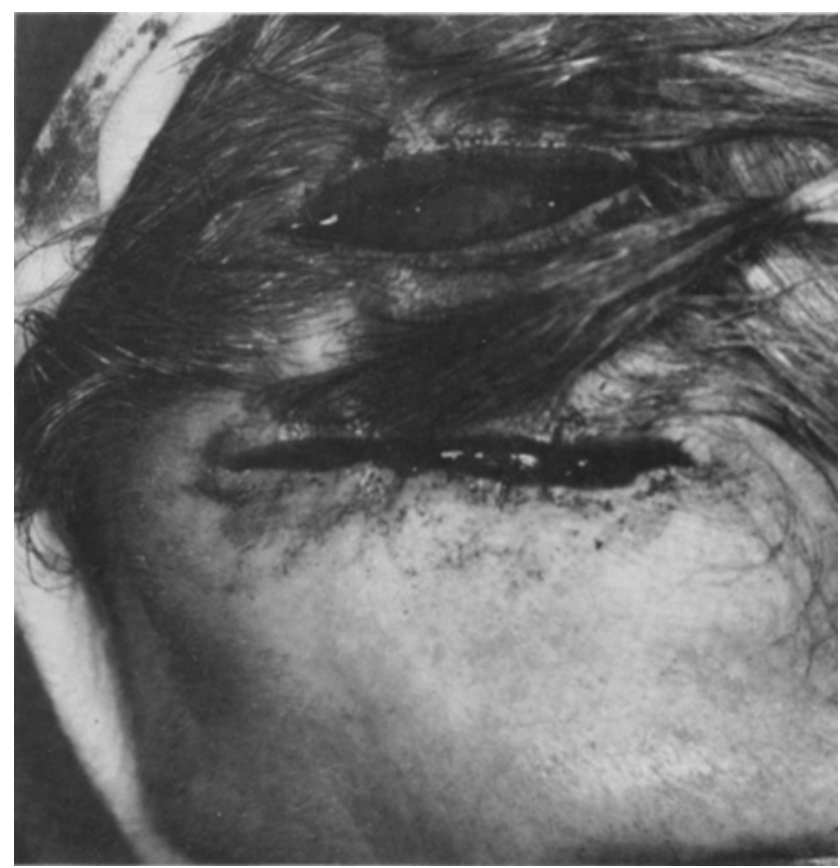

FIGURE 2. The flap has been elevated and advanced anteriorly. The primary defect has been partially closed with subcutaneous sutures.

the width of the wound, corresponding to the amount of tissue movement needed to close the primary defect. The length of this incision corresponds to the long axis of the original defect and parallels the wound. The flap is then further undermined in the subgaleal plane, elevated, and advanced anteriorly. This enables closure of the original defect primarily, maintaining the entire suture line within preexisting skin lines (Fig. 2). The resultant dog-

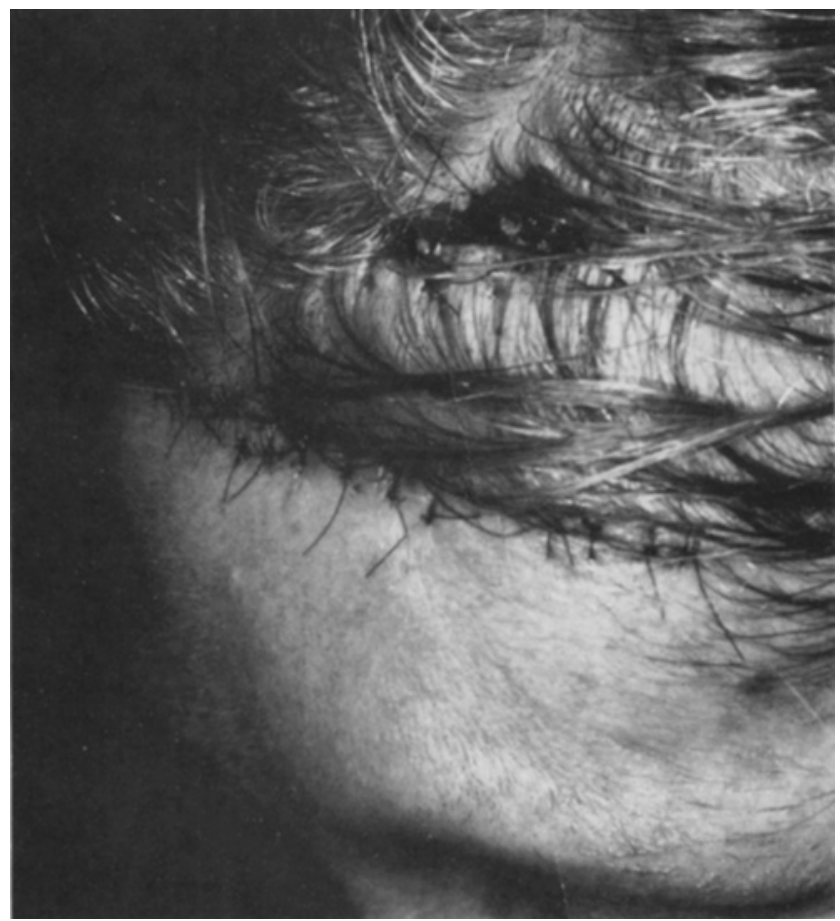

FIGURE 3. Both primary and secondary defects have been closed primarily.

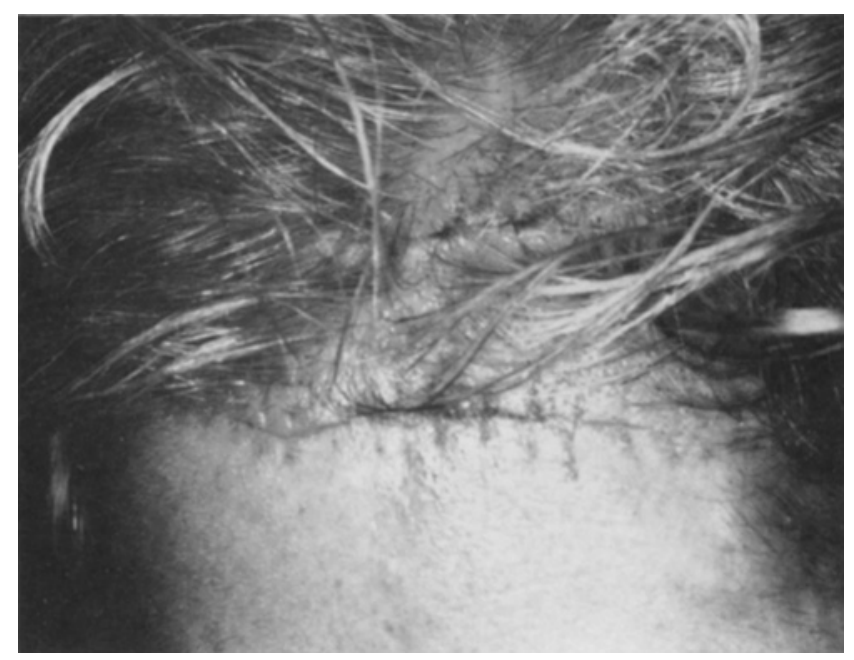

FIGURE 4. One week postoperative. Suture line over primary defect has been placed in existing skin line. Suture line of secondary defect is hidden within the hair.

ears created at the lateral wound margins can then be excised and sutured in the usual fashion, curving the closure to correspond to forehead skin creases (Fig. 3).

The secondary scalp defect, now elliptic in shape, can also be closed primarily. Extensive undermining may be required to facilitate this closure. If necessary, galeotomy or a skin graft may be necessary to cover this defect (Fig. 4). 


\section{DISCUSSION}

Although Grabb and Meyers ${ }^{1}$ briefly discussed this flap in their text, reference to the bipedicle forehead flap is rare. In addition to head and neck wounds, this flap has also been used to close soft tissue defects of the leg and abdomen. ${ }^{3}$ We have found it to be an excellent flap option for forehead defects for several reasons. First, the risk of flap necrosis is small because of the dual blood supply from both pedicles. This "increased" vascularity allows for a greater length/width ratio as opposed to a random single pedicle flap. In our experience, closure of the secondary defect has not been a problem, requiring neither galeal release nor grafting, as some authors suggest. ${ }^{1}$ The suture line is easily covered by the hair in most patients. The visible scar can be further minimized by beveling the skin edges prior to closure, allowing hair to grow through the scar. The length of the visible suture line is minimized because a substantial percentage of tissue movement has occurred within hair-bearing skin, not on the visible forehead. Finally, the slight anterior move- ment of the frontal hairline has not been a cosmetic problem in our patients. In fact, this gives them a somewhat "younger" appearance.

In conclusion, the bipedicle forehead-releasing flap is a safe, technically easy, and cosmetically excellent flap for certain forehead defects. The reconstructive surgeon is able to minimize the length of the visible scar, hide it within existing skin lines, and conceal tissue movement and closure of the secondary defect within the scalp hairline.

\section{REFERENCES}

1. Grabb, W. C., and Meyers, M. B. Skin Flaps. Boston, Little Brown, 1975, pp. 150-151, 165-167.

2. Salibian, H., and Menick, F. J. Bipedicle gastrocnemius musculocutaneous flap for defects of the distal one-third of the leg. Plast. Reconstr. Surg. 70:17-23, 1982.

3. Auda, S. P., and Sindelar, W. F. Bipedicle transverse abdominal cutaneofascial flap for reconstruction of lower thoracic wall defects. J. Surg. Oncol. 15:195-199, 1980. 